\title{
COMPETENCIAS DOCENTES INTERCULTURALES DEL PROFESORADO DE EDUCACIÓN FÍSICA EN ANDALUCÍA (ESPAÑA)
}

\author{
INTERCULTURAL COMPETENCIES OF PHYSICAL EDUCATION TEACHERS IN \\ ANDALUSIA, SPAIN
COMPETENNCIAS DOCENTES INTERCULTURAIS DO PROFESSORADO DE EDUCAÇÃO FÍSICA EM ANDALUZIA (ESPANHA)

José María Fernández Batanero*, José Manuel Aguilar Parra**

Palabras clave:
Emigrantes e
inmigrantes.
Competencia
profesional.
Educación Física y
entrenamiento.

Keywords: Emigrants and immigrants. Professional competence. Physical Education and training.

Palavras chave: Emigrantes e imigrantes. Competência profissional. Educação Física e treinamento.
Resumen: El trabajo versa sobre qué otras competencias debería poseer el profesorado de Educación Física, de educación secundaria, que trabaja con alumnado inmigrante. Para dar respuesta al objetivo planteado adoptamos un diseño de investigación de tipo descriptivo, donde el enfoque de recolección y análisis de datos se ha definido como mixto, en el que se integran técnicas cualitativas (entrevistas en profundidad) y cuantitativas (cuestionario). Los resultados muestran que los docentes que trabajan con alumnado inmigrante poseen un alto nivel en la dimensión de conciencia y actitudinal, sin embargo, se ven claramente limitados en el ámbito de los conocimientos, lo que conlleva a una mayor carencia con respecto a las destrezas y habilidades.

Abstract: This work focuses on the additional competencies needed for secondary-level Physical Education teachers who work with immigrant students. We adopted a descriptive research design in which data collection and its analysis are defined as mixed, as we use both qualitative (in-depth interviews) and quantitative techniques (questionnaire). Results show that teachers who work with immigrant students have high levels in the attitudinal and awareness dimension. However, those teachers are clearly limited in the field of knowledge, therefore, lacking skills and abilities.

Resumo: 0 trabalho centra-se sobre quais outras competências deveria possuir 0 professorado de Educação Física, do ensino médio, que trabalha com alunos imigrantes. Para dar resposta ao objetivo planificado adotamos um desenho de investigação do tipo descritivo, em que o enfoque de recolha e 0 de análises de dados foram definidos como mistos, no qual se integram técnicas qualitativas (entrevistas em profundidade) e quantitativas (questionário). Os resultados mostram que os docentes que trabalham com estudantes imigrantes possuem um alto nível de dimensão de consciência e de atitude, porém, mostram-se claramente limitados no âmbito dos conhecimentos, o que traz consigo uma maior carência no que diz respeito às capacidades e habilidades.
*Universidad de Sevilla. Sevilla, España. E-mail: batanero@us.es

${ }^{* *}$ Universidad de Almería. Almeria, Espanha. E-mail: jmaguilar@ual.es

Recebido em: 18-11-2015 Aprovado em: 06-01-2016

(c) (i) () Licence 


\section{INTRODUCCIÓN}

En las últimas décadas, los movimientos migratorios han ocasionado profundos cambios en la estructura social y educativa de todos los países europeos, y en especial, de España. En el ámbito educativo, la diversidad cultural de la sociedad se refleja en las aulas y, hoy por hoy, es una de las variables que tiene influencia crucial en la convivencia del centro educativo. La presencia de diversas culturas, en el aula, no sólo ha influido en la convivencia, sino también en los resultados del rendimiento académico, especialmente de los alumnos extranjeros. En esta línea, la necesidad de dar respuesta educativa a manifestaciones crecientes de la sociedad actual como el racismo o los conflictos de convivencia entre personas de diferente cultura, se erige como una de las líneas prioritarias del sistema educativo español actual. La Educación Física, como disciplina, no es ajena a esta problemática tal y como lo reflejan los trabajos de Cuevas; Gabaldón, 2005; Cuevas; Fernández Bustos; Pastor, 2009; y en el seno de la misma, se han abierto debates académicos sobre su aportación a los postulados interculturales.

Ahora bien, educar en contextos de diversidad cultural implica definir un modelo de profesor, con al menos, siete competencias básicas: pedagógico-didácticas, de liderazgo, de gestión de grupo y aprendizaje cooperativo, de investigación, interactivas, éticas y competencias sociales (FERNÁNDEZ BATANERO, 2013). Estamos hablando, pues, de un nuevo perfil docente, de un profesorado reflexivo y crítico ante la diversidad cultural.

Así pues, conocedores de esta realidad nos planteamos establecer un análisis de las competencias interculturales que presentan o que deberían estar presentes en el profesorado de Educación Física de Educación Secundaria Obligatoria (ESO) de la Comunidad Autónoma de Andalucía (España), no sólo como facilitadores o mediadores, sino también como factor de cambio. Nuestro trabajo se centra en Andalucía (España), ya que el número de alumnado de origen extranjero matriculado en el curso 2013/2014 fue de 102.174, constituyendo la Comunidad Española con más estudiantes de estas características (CONSEJERÍA DE EDUCACIÓN, 2014).

Ante este contexto, el profesorado de Educación Física (EF) tiene en sus manos una de las mejores herramientas educativas para alcanzar los propósitos interculturales en la escuela. Partiendo de esta premisa, nos hemos planteado las siguientes preguntas que orientan nuestro trabajo: ¿En qué medida el profesorado de educación física se encuentra preparado y formado en competencias interculturales? ¿Qué formación específica han recibido para trabajar con esta tipología de alumnado? ¿Cuál es el grado de satisfacción en el trabajo con alumnado inmigrante? ¿Qué valores desarrollan los centros educativos en relación a la inclusión del alumnado inmigrante?

\section{FUNDAMENTACIÓN TEÓRICA}

A pesar de la unificación que han realizado las instituciones de la Unión Europea (UE) y el Consejo de Europa para consolidar una dimensión intercultural, a la estela de la creciente diversidad cultural relacionada con la migración, todos los países de la UE tienen una gran autonomía en el campo de la educación. Las sociedades europeas se basan en diferentes modelos respecto a la diversidad cultural en la educación, con diferentes consecuencias para los jóvenes según sus experiencias en las escuelas (FAAS; HAJISOTERIOU; PANAYIOTIS, 2014). Así, por ejemplo, en Alemania, Grecia e Irlanda prefieren el término educación intercultural, mientras que en Gran Bretaña y los Países Bajos usan el de multiculturalismo. Y si nos alejamos geográficamente más, Canadá, Estados Unidos y Malasia han trabajado históricamente con el concepto de multiculturalismo. A pesar de las diferencias, el papel de la educación en la integración de los 
inmigrantes y la mejora de la cohesión social parece haber sido aceptada y reconocida a nivel oficial (UNESCO 2009; COUNCIL OF THE EUROPEAN UNION, 2010), así como la percepción de la necesidad de trabajar la diversidad cultural en los centros escolares para la mejor integración de los inmigrantes en la sociedad y, en particular, en el mercado laboral.

La toma de conciencia intercultural se identifica con el conocimiento y la comprensión de las similitudes y diferencias existentes entre el propio universo cultural y el de las comunidades, incluyendo la toma de conciencia de la diversidad regional y social de ambos universos. Además del conocimiento objetivo, la conciencia intercultural engloba la toma de conciencia del modo en que cada comunidad es contemplada desde la óptica de los demás, frecuentemente caracterizados por los estereotipos. Por último, las destrezas y habilidades interculturales remiten a cuatro capacidades: la capacidad de establecer relaciones entre la cultura de origen y la extranjera; la sensibilidad cultural y la capacidad de emplear estrategias variadas para establecer contacto con personas de otras culturas; la capacidad de desempeñar el papel de intermediario cultural entre la propia cultura y la extranjera y de resolver situaciones de conflicto y malentendidos culturales; y la capacidad para superar estereotipos (PARICIO, 2004). Así pues, se constata que la interculturalidad supone una reflexión profunda en torno a las oportunidades que nos ofrece la diversidad cultural como elemento de intercambio fructífero de valores y actitudes, rompiendo prejuicios e iniciando espacios de interrelación e intercambio, creando así lazos e interdependencias entre los grupos culturales diversos que conviven en una misma realidad escolar (LEIVA OLIVENCIA, 2008).

Son abundantes los trabajos que relacionan de forma positiva la Educación Física y la interculturalidad, ya que constituye un medio imprescindible para el desarrollo de valores y actitudes (BEHNKE; ROTH, 2002). En el contexto español, estudios como los de López; Pérez; Monjas (2007) sobre la integración del alumnado, en el que participan los profesores, obtienen evidencias que indican que la mayoría de los participantes afirman el hecho de que la EF potencia la integración de los inmigrantes. Por otro lado, Valero; Gómez (2007) exponen que la EF es una materia en la que se trabajan aspectos que contribuyen a la integración escolar del alumnado inmigrantes, bien sea por sus características específicas o por la adecuación de sus contenidos. Es una materia motivadora que educa en valores y que, a través de los juegos y actividades, favorece el rechazo de actitudes discriminatorias al potenciar la igualdad y el respeto al diferente (RUIZ VALDIVIA et al., 2012). Ahora bien, a pesar de ello, también existen trabajos que coinciden en subrayar el vacio científico que actualmente existe sobre el interculturalismo y la Educación Física (CHOI; CHEPYATOR-THOMSON, 2011; GRIMMINGER, 2011; LÓPEZ PASTOR, 2012).

Es en este contexto de convivencia escolar donde la figura del docente es esencial para el fomento y desarrollo de la interculturalidad, por lo que sus actitudes, estrategias e interrelaciones en la tarea educativa deben reflejar su compromiso ciudadano de transformar el contexto social y escolar. Estamos hablando, pues, de un nuevo perfil docente, de un profesorado reflexivo y crítico ante la diversidad cultural, es decir, un profesional abierto al diálogo, a la deliberación grupal, a la escucha de opciones distintas a la suya. La formación del profesorado se convierte en un instrumento de primer orden para el desarrollo del diálogo intercultural (SALES, 2012), favoreciendo la construcción del conocimiento por sí mismo, de forma crítica y reflexiva. En esta línea, han sido numerosos los autores, tanto a nivel nacional como internacional, que en los últimos años han estudiado cómo debería ser la formación intercultural del profesorado en general (JOKIKOKKO, 2005; PERRY; SOUTHWELL, 2011; PINYA; MUNTANER, 2014; TAROZZI, 2014), y del profesorado de educación física en particular (FLORES; PRAT; SOLER, 2014). 
Resulta interesante resaltar la ausencia de propuestas formativas documentadas destinadas a la formación intercultural del futuro profesorado especialista en Educación Física en el contexto español, mientras que a nivel internacional existen diferentes propuestas formativas como la que destaca la experiencia de Burden; Hodge; O'bryant; Harrison (2004) o la de GiesStuber (2008), donde se describen un ambicioso proyecto formativo sobre el Desarrollo de la competencia intercultural a través del deporte. Otras experiencias formativas destacables son las de Bender-Szymanski (2012), Gies-Stuber (2008) Y Grimminger (2011) en Alemania; y la de Benn; Dagkas (2006) en la Universidad de Birmingham (Reino Unido).

\section{METODOLOGÍA}

El objetivo principal del estudio ha sido la obtención de información sobre qué otras competencias debería poseer el profesorado de Educación Física de educación secundaria que trabaja con alumnado inmigrante, en la Comunidad Autónoma de Andalucía (España). Para ello, los informantes han sido los propios profesores de educación física y profesorado universitario que imparten en la especialidad de educación física.

El diseño de la investigación se sitúa desde una perspectiva de investigación de estudio de caso de carácter descriptivo, a partir del cual el enfoque de recolección y análisis de datos se ha definido como mixto, en el que se integran técnicas cualitativas y cuantitativas.

El cuestionario tipo Likert dirigido al profesorado es una adaptación del utilizado por Murua-Carton, et al. (2012) en el contexto del País Vasco Español y aplicado en el nivel de educación infantil y primaria. Nuestro cuestionario, una vez adaptado y validado, quedó constituido por 40 ítems distribuidos en 4 bloques: consciencia, actitud, conocimiento, y Destreza-Habilidades. En ellos, se describen determinadas características que debe presentar el profesorado que trabaja con alumnado inmigrante en la etapa de educación secundaria. El cuestionario fue remitido al profesorado mediante una herramienta de cuestionario online, que puede consultarse en la siguiente dirección web: https://docs.google.com/forms/d/1bHDqA-tpy LuRKYJFu5PsuAwOvv7Q8kCf52jvNPzvJtw/viewform?embedded=true

Cuadro 1 - Distribución de los ítems por dimensiones

\begin{tabular}{|c|c|c|}
\hline Dimensión & Definición & Ítems \\
\hline Conciencia & $\begin{array}{l}\text { Conocimiento que el individuo tiene de sus actos, de sí mismo o de sus } \\
\text { propias reflexiones } \\
\text { Esta dimensión se relaciona con aspectos tan diversos como la cultura, la } \\
\text { empatía, la capacidad de escuchar, la posibilidad de enriquecimiento mutuo } \\
\text { entre el alumnado autóctono y el profesorado con el alumnado inmigrante. }\end{array}$ & $\begin{array}{l}1,2,3,4,5,6,7,8 \\
9,10,11\end{array}$ \\
\hline Actitud & $\begin{array}{l}\text { Disposición de ánimo de cada individuo. Se relaciona con problemas deri- } \\
\text { vados de actitudes racistas o xenófobas }\end{array}$ & $\begin{array}{l}12,13,14,15,16 \\
17,18,19,20,21\end{array}$ \\
\hline Conocimiento & $\begin{array}{l}\text { Nociones que posee el individuo respecto de un tema dado. Relacionado } \\
\text { con al grado de información sobre aspectos culturales autóctonos y } \\
\text { foráneos, el conocimiento de ciertos términos de la lengua del alumna- } \\
\text { do o la dinámica de trabajo grupal que pudiera facilitar la integración } \\
\text { del alumno/a de nueva incorporación. }\end{array}$ & $\begin{array}{c}22,23,24,25,26, \\
27,28,29,30\end{array}$ \\
\hline $\begin{array}{l}\text { Destreza y } \\
\text { Habilidades }\end{array}$ & $\begin{array}{l}\text { Capacidad para realizar algún trabajo. Concerniente con las facilidades o } \\
\text { dificultades con las que se encontraba habitualmente el/la educador/a } \\
\text { cuando intentaba mejorar el rendimiento del alumnado inmigrante, su } \\
\text { capacidad de integración de las nuevas culturas o su participación a la } \\
\text { hora de la creación de espacios de convivencia }\end{array}$ & $\begin{array}{l}31,32,33,34,35 \\
36,37,38,39,40\end{array}$ \\
\hline
\end{tabular}


La población de profesores y profesoras de educación física en septiembre de 2012, que imparten docencia en Andalucía (España) era de 1.563, repartidos en 847 centros. Del total de profesores y profesoras a los que se les envió el cuestionario, respondieron 254 , lo que representa el $16,25 \%$.

Para la validación del cuestionario (validez de contenido), se seleccionó a un grupo de expertos compuesto por 14 profesores universitarios especialistas en atención a la diversidad, y ocho profesores no universitarios con experiencia en el alumnado inmigrante. Para su selección se utilizó el procedimiento de "Coeficiente de competencia experta" o "Coeficiente K" obtenido mediante la aplicación de la siguiente fórmula: $K=1 / 2(K c+K a)$, donde $K c$ es el "Coeficiente de conocimiento" o información que tiene el experto acerca del tema o problema planteado; y Ka es el denominado "Coeficiente de argumentación" o fundamentación de los criterios de los expertos (CABERO; FERNÁNDEZ BATANERO; ORTA, 2015). En nuestro caso, el coeficiente $K$ fue superior a 0,8 en 18 de los 22 expertos seleccionados, denotando ello un grado de competencia muy aceptable. Las estimaciones de los expertos se realizaron en sucesivas rondas, anónimas, al objeto de tratar de conseguir consenso, pero con la máxima autonomía por parte de los participantes (método Delphi).

Cuadro 2 - Ejemplo de preguntas a cumplimentar por el experto

¿Ha impartido a lo largo de su vida profesional asignatura/contenidos/materias relacionadas con la atención a la diversidad del alumnado? ( ) SI ( ) No ¿Ha dirigido o participado en alguna investigación relacionada con aspectos de la educación multicultural o intercultural? ( ) SI ( ) No

¿Ha realizado o participado en alguna publicación relacionada con la educación intercultural? ( ) SI ( ) No

Marque en la casilla el grado de conocimiento que usted posee acerca de la temática del alumnado inmigrante.

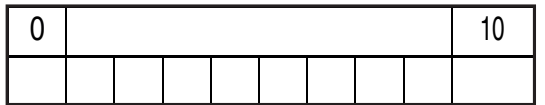

Fuente: Elaboración propia

El cálculo de la fiabilidad del cuestionario se realizó mediante el coeficiente de fiabilidad conocido como Alpha de Cronbach, donde se han obtenidos valores bastantes elevados en lo que se refiere a su conjunto $(0,896)$. Para asegurar más aún la fiabilidad del instrumento, se le aplicaron otras pruebas: prueba de las dos mitades (valor de $\alpha$ para la primera parte: 0,889; valor de $\alpha$ para la segunda parte: 0,882; coeficiente de Spearman-Brown: 0,857, prueba KMO (índice significativo de 0,89 , próximo a la unidad), obteniéndose un nivel elevado de confiablidad. Para el análisis de los datos, se utilizó el programa SPSS 15.0.

Con respecto a las entrevistas en profundidad, decir que la selección de los entrevistados se realizó teniendo en cuenta criterios de "significatividad", o, lo que es lo mismo, la relevancia de las personas por su relación con los objetivos de la investigación y a la población a la que esta se refiere. Nuestros informantes claves fueron 14 profesores universitarios que imparten docencia en el grado de la Actividad Física y del Deporte de tres universidades andaluzas (Universidad de Sevilla, Universidad de Granada y Universidad Pablo de Olavide). Para el tratamiento y análisis de la información se optó por la herramienta informática de análisis cualitativo Nudist Vivo 7.0. 


\section{PRINCIPALES RESULTADOS}

Apoyados en la base empírica de las percepciones y opiniones de los informantes, intentaremos reflexionar sobre los primeros puntos de encuentro de esta investigación.

\subsection{Cuestionario}

El profesorado de educación secundaria encuestado corresponde en $58 \%$ a hombres y $42 \%$ a mujeres. Atendiendo a su situación laboral el $61 \%$ son funcionarios y el 39\% interinos y contratados. En relación con la experiencia laboral, el 33\% tiene una experiencia entre 11 y 15 años, el $42 \%$ entre 15 y 25 años, el $12 \%$ entre 5 y 10 años y el $13 \%$ más de 25 años (Cfr. Tabla 1).

Tabla 1 - Clasificación de las competencias emprendedoras en función de la puntuación media obtenida en el cuestionario

\begin{tabular}{|c|c|c|c|}
\hline El profesor debe & Media & $\begin{array}{l}\text { Desviación } \\
\text { Típica }\end{array}$ & Asimetría \\
\hline $\begin{array}{l}\text { Ser consciente de la necesidad de ponerse en el lugar de la } \\
\text { persona de la otra cultura para poder entenderla }\end{array}$ & 8,90 & 2,000 & 0,027 \\
\hline $\begin{array}{l}\text { Saber las necesidades que se les presentan a las personas } \\
\text { inmigrantes a su llegada a los centros }\end{array}$ & 8,89 & 2,005 & 0,032 \\
\hline $\begin{array}{l}\text { Intentar eliminar del centro cualquier expresión de racismo o } \\
\text { xenofobia }\end{array}$ & 8,88 & 1,833 & 0,028 \\
\hline $\begin{array}{l}\text { Ser consciente del enriquecimiento que producen las relaciones } \\
\text { entre personas de diversas culturas }\end{array}$ & 8,38 & 2,010 & 0,035 \\
\hline $\begin{array}{l}\text { Ser consciente que los estereotipos pueden afectar a la } \\
\text { autoestima y autoconcepto }\end{array}$ & 8,35 & 2,095 & 0,021 \\
\hline $\begin{array}{l}\text { Tener conocimiento de las consecuencias que se derivan de } \\
\text { actitudes racistas y xenófobas }\end{array}$ & 8,33 & 2,165 & 0,128 \\
\hline $\begin{array}{l}\text { Ser consciente de la importancia de dar ejemplo cuando se } \\
\text { producen incidentes debidos a diferencias }\end{array}$ & 8,32 & 1,907 & 0,062 \\
\hline $\begin{array}{l}\text { Conocer los valores, actitudes, tradiciones, creencias y } \\
\text { costumbres de la propia cultura }\end{array}$ & 8,31 & 1,677 & 0,044 \\
\hline $\begin{array}{l}\text { Ser capaz de crear un clima de convivencia basado en el respeto } \\
\text { mutuo y la solidaridad entre el alumnado }\end{array}$ & 8,08 & 1,998 & 0,023 \\
\hline $\begin{array}{l}\text { Asumir un papel activo para evitar conflictos por razones culturales } \\
\text { en el centro }\end{array}$ & 8,01 & 2,077 & 0,133 \\
\hline Conocer la influencia del plurilingüismo en el desarrollo educativo & 7,62 & 2,099 & 0,245 \\
\hline $\begin{array}{l}\text { Ser capaz de mejorar el rendimiento del alumnado inmigrante en } \\
\text { la(s) lengua(s) del centro educativo }\end{array}$ & 7,55 & 2,098 & 0,222 \\
\hline Fomentar el éxito escolar del alumnado inmigrante & 7,74 & 2,067 & 0,378 \\
\hline $\begin{array}{l}\text { Estar dispuesto a trabajar para superar las resistencias que surgen } \\
\text { en la convivencia entre culturas }\end{array}$ & 7,54 & 1,956 & 0,481 \\
\hline $\begin{array}{l}\text { Asumir que se produzcan cambios en su trabajo como } \\
\text { consecuencia de la incorporación del alumnado inmigrante }\end{array}$ & 7,41 & 2,009 & 0,465 \\
\hline $\begin{array}{l}\text { Ser consciente de la influencia de la cultura de origen en los } \\
\text { grupos inmigrantes }\end{array}$ & 6,99 & 2,008 & 0,455 \\
\hline $\begin{array}{l}\text { Tener información del país de origen (educación, política, arte, } \\
\text { religión...) para trabajar eficazmente con alumnado inmigrante }\end{array}$ & 6,85 & 1,999 & 0,407 \\
\hline
\end{tabular}


Continuación da tabla 1...

Ser consciente de la importancia que tiene la lengua en una cultura

6,4

2,001

0,509

Diseñar y poner en marcha nuevas experiencias de aprendizaje que reconozcan la diversidad cultural como parte de los contenidos del curso

Saber algunas palabras y expresiones de las lenguas de origen del alumnado extranjero para facilitar la comunicación

Conocer las propias limitaciones culturales

Ser consciente de la influencia de su propia cultura

Ser consciente de la necesidad de colaborar con otros profesionales para abordar los temas interculturales

Organizar actividades que enriquezcan la convivencia entre las diferentes culturas

Ser consciente de la necesidad de escuchar abiertamente

Conocer las barreras que surgen al incorporarse prácticas derivadas de otras culturas en el funcionamiento del centro educativo (calendario escolar, horarios, comedor, lenguas, indumentaria...)

Ser capaz de analizar las necesidades asociadas a la convivencia intercultural en el centro educativo

$6,40 \quad 1,954 \quad 0,576$

$6,28 \quad 2,278 \quad 0,487$

$6,24 \quad 2,189 \quad 0,324$

$6,22 \quad 2,001 \quad 0,487$

$6,22 \quad 1,976 \quad 0,577$

$6,21 \quad 2,173 \quad 0,487$

$6,20 \quad 1,765 \quad 0,521$

$6,19 \quad 2,084 \quad 0,564$

$6,18 \quad 1,998 \quad 0,249$

Conocer dinámicas de grupo que favorezcan la convivencia entre culturas

$6,18 \quad 1,915 \quad 0,598$

Ser capaz de obtener información sobre una cultura determinada

$6,16 \quad 2,100 \quad 0,612$

Fomentar los contactos con familiares y asociaciones de inmigrantes para entender mejor las situaciones que se puedan presentar en el centro

Ser capaz de reflexionar y evaluar sobre las acciones que ha desarrollado para mejorar

Estar dispuesto a trabajar en la elaboración y puesta en marcha de planes y programas específicos para la integración de alumnos inmigrantes

Estar dispuesto a trabajar con inmigrantes fuera del contexto del centro educativo

Ser capaz de pedir consejo a líderes espirituales de la comunidad del alumnado

Adaptar los recursos humanos, materiales y medios para la mejora de la práctica educativa con alumnado inmigrante

Ser consciente del impacto que mi estilo de comunicación tiene sobre el alumnado

Ser consciente de que los malentendidos pueden surgir en las relaciones entre personas de distintas culturas

Ser capaz de enviar y recibir mensajes verbales y no verbales de manera apropiada

Crear espacios y momentos donde el alumnado inmigrante pueda practicar la lengua de origen

$6,15 \quad 2,063 \quad 0,694$

$6,14 \quad 2,103 \quad 0,367$

$6,14 \quad 2,088 \quad 0,488$

$6,12 \quad 2,098 \quad 0,567$

$6,11 \quad 1,988 \quad 0,582$

$6,11 \quad 2,273 \quad 0,622$

$5,98 \quad 2,123 \quad 0,632$

$5,93 \quad 1,991 \quad 0,611$

$5,71 \quad 2,200 \quad 0,620$

$5,70 \quad 1,978 \quad 0,624$

Conocer elementos culturales (verbales y no verbales) para enriquecer las relaciones interculturales

$5,69 \quad 2,256 \quad 0,627$


Todas las medias de las cuatro dimensiones sobrepasan la media de las medias $(6,89)$, como puede observarse en el grafico 1, excepto destrezas y habilidades (6,41) (Cfr. Gráfico 1).

Grafico 1 - Puntuación media por dimensión

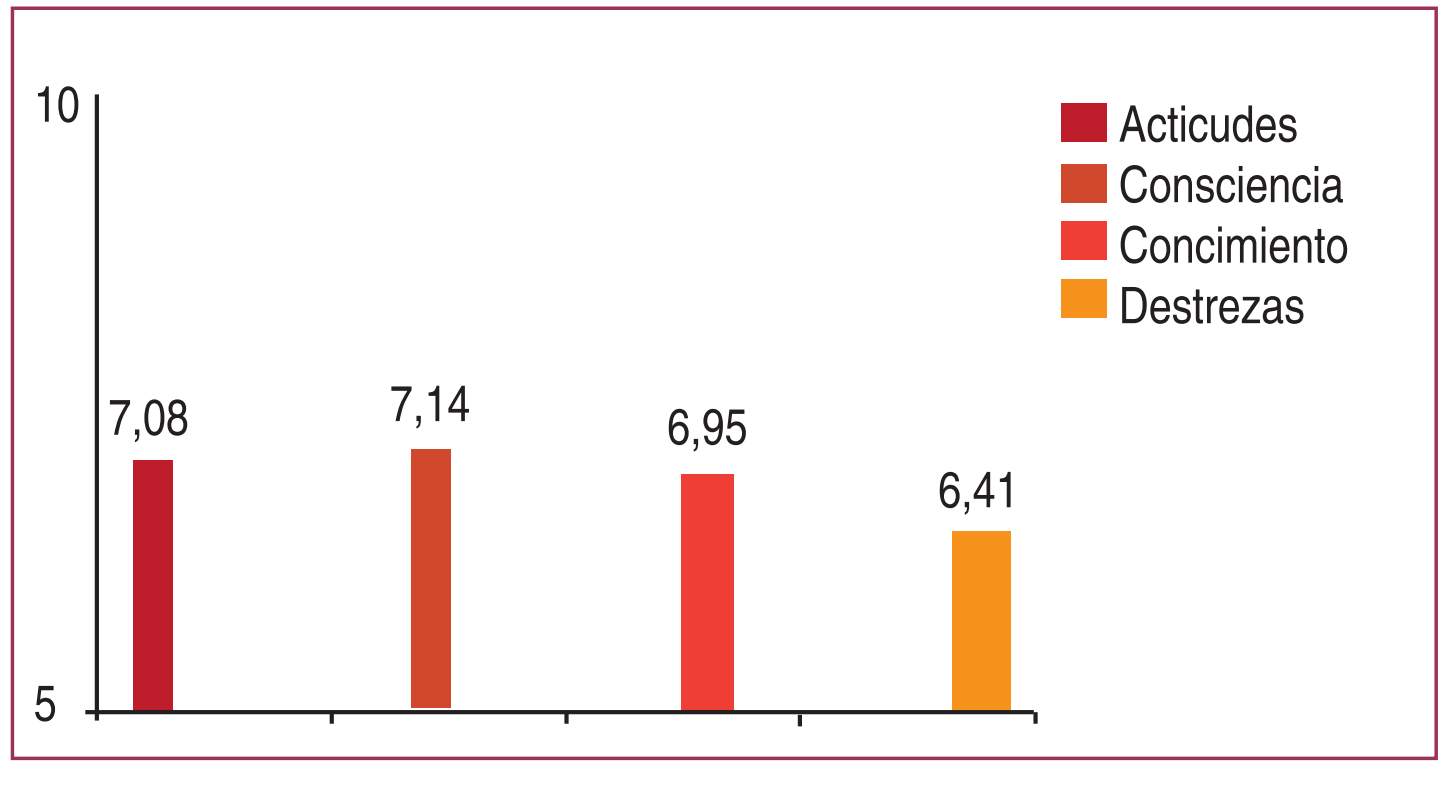

Fuente: Elaboración propia.

En este sentido, podemos afirmar que los datos obtenidos a nivel general muestran con claridad que el profesorado que trabaja con alumnado inmigrante es consciente de la labor que desempeña, mostrando a la vez actitudes favorables ante esa diversidad de alumnado y aportando conocimiento acerca de esta tipología de alumnado. Ahora bien, los mayores problemas se presentan en relación con las destrezas y los conocimientos.

En relación con los datos aportados por la tabla 1, el profesorado que trabaja con alumnado inmigrante cree que debe ante todo ser consciente de la necesidad de ponerse en el lugar de la persona de la otra cultura para poder entenderla (puntuación media de 8,90; Desviación Típica "DT": 1,890). Este ítem posee el valor de desviación típica más bajo, lo que refleja la escasa dispersión de las respuestas. Saber las necesidades que se les presentan a las personas inmigrantes a su llegada a los centros (puntuación media 8,89; DT: 2,005).

Las posibles dificultades u obstáculos pueden surgir a la hora de valorar los conocimientos y las destrezas que debería poseer el profesorado, así, nos encontramos con que de seis ítems pertenecientes al grupo de los conocimientos, están por debajo de la media de las medias de los ítems $(6,89)$ : "Tener información del país de origen (educación, política, arte, religión...) para trabajar eficazmente con alumnado inmigrante." (6,85); "Saber algunas palabras y expresiones de las lenguas de origen del alumnado extranjero para facilitar la comunicación" (6,28); "Conocer las propias limitaciones culturales" (6,24); "Conocer las barreras que surgen al incorporarse prácticas derivadas de otras culturas en el funcionamiento del centro educativo (calendario escolar, horarios, comedor, lenguas, indumentaria...)" $(6,19)$; "Conocer dinámicas de grupo que favorezcan la convivencia entre culturas" $(6,18)$; "Conocer elementos culturales (verbales y no verbales) para enriquecer las relaciones interculturales" $(5,69)$.

Pero los mayores obstáculos se presentan con la dimensión de las destrezas y habilidades. En este sentido, decir que siete ítems están por debajo de la media de las 
medias de los ítems (6,89): "Ser capaz de analizar las necesidades asociadas a la convivencia intercultural en el centro educativo" $(6,18)$; "Ser capaz de obtener información sobre una cultura determinada" $(6,16)$; "Ser capaz de reflexionar y evaluar sobre las acciones que ha desarrollado para mejorar" (6,14); "Ser capaz de pedir consejo a líderes espirituales de la comunidad del alumnado" (6,11); "Adaptar los recursos humanos, materiales y medios para la mejora de la práctica educativa con alumnado inmigrante" $(6,11)$; "Ser capaz de enviar y recibir mensajes verbales y no verbales de manera apropiada" $(5,71)$ y "Crear espacios y momentos donde el alumnado inmigrante pueda practicar la lengua de origen" $(7,70)$. Con base en estos resultados, podemos detectar claramente dos tipos de problemas a los que se enfrenta el profesorado: por un lado, la poca importancia que los docentes le otorgan al conocimiento de la comunicación verbal, incidiendo posiblemente ello en la creación de espacios donde estas personas tengan unos espacios o momentos donde puedan prácticas la lengua de origen. Ahora bien, también debemos destacar la importancia que se le concede a la capacidad de reflexión y evaluación con objeto de mejora $(6,14)$, incidiendo ello en la capacidad para analizar las necesidades asociadas a la convivencia intercultural $(6,18), y$ por consiguiente en la mejora del rendimiento de este alumnado $(7,55)$.

En relación con el nivel de Consciencia que presentan los profesores y profesoras, hay que destacar que a pesar de que sólo cinco ítems se sitúan por encima de la media de las medias de los ítems, éstos ocupan los primeros puestos: "Ser consciente de la necesidad de ponerse en el lugar de la persona de la otra cultura para poder entenderla" $(8,90)$, "Ser consciente del enriquecimiento que producen las relaciones entre personas de diversas culturas" $(8,38)$, "Ser consciente que los estereotipos pueden afectar a la autoestima y autoconcepto" $(8,35)$ y "Ser consciente de la importancia de dar ejemplo cuando se producen incidentes debidos a diferencias" $(8,32)$, ocupaban los primeros siete puestos.

En el análisis de las aptitudes del profesorado, nos encontramos con los resultados que cuatro de los nueve ítems de esta dimensión se encuentran por encima de la media, obteniendo puntuaciones muy satisfactorias, como es el caso de "Intentar eliminar del centro cualquier expresión de racismo o xenofobia" (8,88, DT: 1,844), que obtiene la tercera posición. Le siguen "Fomentar el éxito escolar del alumnado inmigrante" (7,74); "Estar dispuesto a trabajar para superar las resistencias que surgen en la convivencia entre culturas" $(7,54)$ y "Asumir que se produzcan cambios en su trabajo como consecuencia de la incorporación del alumnado inmigrante" $(7,41)$. De los ítems menos valorados con encontramos con "Estar dispuesto a trabajar con inmigrantes fuera del contexto del centro educativo" $(6,12)$, así como la disposición para trabajar en la elaboración y puesta en marcha de planes y programas específicos $(6,14)$. También podemos destacar el ítems "Fomentar los contactos con familiares y asociaciones de inmigrantes para entender mejor las situaciones que se puedan presentar en el centro" $(6,15)$ con una media por debajo de las que no ha servido de referencia.

\subsection{Entrevistas}

Las entrevistas giraban en torno a cuatro grandes dimensiones: formación del profesorado; necesidades del alumnado inmigrante; recursos y competencias docentes para la interculturalidad. El análisis de los discursos confirma y amplia los resultados obtenidos en otras investigaciones (MURUA-CARTON, et al., 2012; DÍEZ GUTIÉRREZ, 2014). Con respecto a la 
primera dimensión, los entrevistados consideran que el profesorado de educación física posee una moderada formación sobre la atención a la diversidad, especialmente con el alumnado inmigrante.

Hace unos años no estábamos tan concienciados con la presencia de alumnado inmigrante. La verdad que el profesorado universitario es mucho más sensible a este fenómeno. Nuestros alumnos en su formación inicial reciben formación en materia de atención a la diversidad del alumnado. (ENT. 2).

Un gran porcentaje del profesorado universitario es consciente de las necesidades del alumnado inmigrante e intenta poner de su parte para lograr su integración e inclusión en el centro educativo.

Yo como profesor me pongo muchas veces en la piel del alumnado. El profesorado de de mi universidad es consciente de la importancia de la Educación Física como medio de inclusión educativa. (ENT. 6).

Esa toma de conciencia del profesorado lleva consigo el despliegue de una serie de actitudes acordes con la atención a la diversidad, y que en la mayoría de los casos contribuyen a fomentar un clima favorable de convivencia. Ahora bien, existen casos en los que el profesorado debido a su falta de formación se ve impotente, siendo una causa de ello la ausencia de profesorado de apoyo.

En relación a las necesidades del alumnado inmigrante, decir que los entrevistados hacen fundamentalmente hincapié en los "problemas" que les supone al profesorado y al funcionamiento general del centro, la presencia de esta tipología de alumnado. Consideran que el alumnado inmigrante y sus características no constituyen un hándicap para el desarrollo del proceso de enseñanza-aprendizaje y, en algunos casos, afectando a la convivencia.

\footnotetext{
El profesor que se encuentra formado en relación a la atención a la diversidad es consciente de la importancia de esta asignatura para resolver los posibles de integración. El desconocimiento de nuestra lengua en las clases de Educación Física no constituye un hándicap. El deporte es un instrumento de integración universal. (ENT. 9).
}

En los entrevistados existe una sensación generalizada de abandono por parte de la Administración Educativa. La mayoría del profesorado coincide en que desde la Administración se puede hacer más, fundamentalmente en el ámbito de la formación intercultural para atender el gran desafío que supone la multiculturalidad y los problemas que genera la diversidad cultural, tanto a nivel del profesorado, alumnado y centro educativo. En esta línea, la Administración educativa debería de potenciar y dotar de medios a los centros educativos para el desarrollo de planes de acogida, y legislar sobre la figura de un responsable en materia intercultural. Así mismo, se pone especial énfasis en el desarrollo de protocolos especiales para el alumnado inmigrante, de forma que se favorezca el proceso de inscripción.

En relación a la dimensión sobre competencias docentes los entrevistados hacen mención a la importancia de tener una actitud favorable hacia el alumnado inmígrate. En este sentido, se manifiestan de una fuerte carga de valores como requisito imprescindible para la mediación de los procesos educativos de este alumnado, y que se agrupan en torno al desarrollo de la competencia social (comprensión, amistad, respeto, responsabilidad, etc.).

Para ejercer la docencia en estos contextos de diversidad, es necesario la 
comprensión de puntos de vista diferentes...Yo diría que más que competencias interculturales hablaría de carga de valores [...]. (ENT. 6).

Los entrevistados consideran que la inclusión requiere un profesorado coherente, es decir, fiel a sus principios y sus creencias. Docentes que crean en lo que están haciendo y en lo hay que hacer. Un docente transmisor de valores. En este sentido, se apuesta por el desarrollo de competencias éticas para desarrollar valores, como aspecto esencial que debe tener todo docente en su formación.

\section{CONCLUSIONES}

Los resultados del estudio permitieron llegar a las siguientes conclusiones:

El profesorado de Educación Física presenta un alto nivel en la dimensión de conciencia y en la dimensión actitudinal, en relación con la actividad que desarrollan en los respectivos centros educativos. Sin embargo, se ve claramente delimitado en el ámbito de los conocimientos, lo que conlleva a una mayor carencia con respecto a las destrezas y habilidades. Este aspecto viene a ratificar una cierta falta de preparación del profesorado que trabaja con este tipo de alumnado, a pesar de ello, muestra una actitud muy favorable hacia su trabajo. Aspectos coincidentes con estudios como los realizados por Murua-Carton et al. (2012), con el profesorado de educación primaria. El profesorado no se considera formado suficientemente en el desarrollo de competencias interculturales, desempeñando su función de forma satisfactoria debido al desarrollo de estrategias docentes por iniciativas propias.

Los valores que se deben desarrollar en la formación inicial del profesorado de Educación Física debe enfatizar el componente social, incorporando la dimensión moral y emocional en los procesos, sin restar eficacia a éstos y a los resultados obtenidos. Este trabajo más humanizado y más creativo, responde mejor a las necesidades actuales que conlleva el alumnado inmigrante, apostando por el desarrollo de competencias éticas que generen nuevos valores, rompiendo prejuicios e iniciando espacios de interrelación e intercambio (LEIVA OLIVENCIA, 2008).

El profesorado de Educación Física destaca sobre todo los problemas de comunicación que le supone el desconocimiento de la lengua del país receptor y la experiencia, casi siempre traumática, de la inmigración y el desarraigo. Se percibe un gran interés por parte del profesorado para que los niños extranjeros aprendan el idioma español lo más rápidamente posible con el fin de incorporarse a las actividades que se desarrollan en el aula y que están respondiendo a una determinada cultura (costumbres, valores, tradiciones, lengua, etc. españolas). Hallazgos coincidentes con otros estudios en el contexto español (PARICIO, 2004, LEIVA OLIVENCIA, 2008).

\section{REFERENCIAS}

BEHNKE, Ute; ROTH, Hans-Jochim. Inmigración y escuela: Experiencias de integración en Hamburgo. Apunts. Educación Física y Deportes, Barcelona, n.68, p. 50-56, 2002.

BENDER SZYMANSKI, Dorothea. Assimilation, segregation, or integration? A teaching project examining approaches to religious and ideological diversity in the classroom. Intercultural Education, Abingdon, v. 23, n. 4, p. 325-340, 2012. 
BENN, Tansin; DAGKAS, Symeon. Incompatible? Compulsory mixed-sex physical education initial teacher training (PEITT) and the inclusion of Muslim women: a case-study on seeking solutions". European Physical Education Review, London, v. 12, n. 2, p. 181-200, 2006.

BURDEN, Joe; HODGE, Samuel; O'BRYANT, Camille; HARRISON, Louis. From colorblindness to intercultural sensitivity: infusing diversity training in PETE programs. Quest, Abingdon, v. 56, n. 2, p.173-189, 2004.

CABERO, Julio; FERNÁNDEZ BATANERO, José María; ORTA, Isabel. Dificultades de Lectura y Escritura en Alumnos con Nacimiento Prematuro. Construcción de un Instrumento de Diagnóstico. REVALUE. Revista de Evaluación Educativa, Huixquilucan, v. 4, n. 1, 2015. Disponible en: <http:// revalue.mx/revista/index.php/revalue/article/view/124>. Acceso: 13 sept. 2015.

$\mathrm{CHOI}$, Wonseok; CHEPYATOR-THOMSON, Rose. Multiculturalism in teaching physical education: a review of U.S. based literature. Journal of Research, Abingdon, v. 6, n. 2, p. 14-20, 2011.

CONSEJERÍA DE EDUCACIÓN. La educación en Andalucía. Curso 2013-2014. Sevilla: Junta de Andalucía. Disponible en: <http://www.iseandalucia.es/c/document library/get file?uuid=286cd0dc-56bd-4128-95af-6f038ec66518\&groupld=10137. Acceso: 20 ago. 2015.

COUNCIL OF THE EUROPEAN UNION. Europe 2020: A strategy of smart, sustainable and inclusive growth. Bruselas: European Commission, 2010.

CUEVAS, Ricardo; FERNÁNDEZ BUSTOS, Juan Gregorio; PASTOR, Juan Carlos. Educación Física y educación interculrural: Análisis y propuestas. Ensayos, Revista de la Facultad de Educación de Albacete. Albacete, 2009. Disponible en: <http://www.uclm.es/ab/educacion/ ensayos. >. Acceso: 1․ sept. 2015.

CUEVAS, Ricardo; GABALDÓN, Juan Antonio. Educación Física de aquí y allí: la diferencia nos enriquece; proyecto de innovación e investigación en la acción educativa. In: CONGRESO INTERNACIONAL AÑO DEL DEPORTE Y LA EDUCACIÓN FÍSICA. Cuenca, 2005. Disponible en: http://deporteparatodos.com/imagenes/documentacion/ficheros/20060613004542Area4 Comunicaciones.pdf . Acceso en: 6 ago. 2015.

DÍEZ GUTIÉRREZ, Enrique Javier. La práctica intercultural en secundaria. Revista de Educación, Madrid, n. 363, p. 12-34, 2014. Disponible en:<http://dx.doi.org/10.4438/1988592X-2011-363-168>. Acceso en: 10. sept. 2015.

FAAS, Daniel; HAJISOTERIOU, Christina; PANAYIOTIS, Angelides. Intercultural education in Europe: policies, practices and trends. Bristish Educational Research Journal, Abingdon, v. 40, n. 2, p. 300-318, 2014.

FERNÁNDEZ BATANERO, José María. Competencias docentes y educación inclusiva. REDIE, Ensenada, v.15, n. 2, 2013. Disponible en: <http://redie.uabc.mx/index.php/redie/article/ view/445/573>. Acceso: 18 ago. 2015.

FLORES, Gonzalo; PRAT, María; SOLER, Susana. La voz del profesorado de educación física sobre su formación académica ante la realidad multicultural: análisis de la situación y propuestas de mejora. Revista Electrónica Interuniversitaria de Formación del Profesorado, Granada, v. 17, n. 2, p. 183-199, 2014.

GIES-STUBER, Petra. Travelling trough Europe. Estrangement and return. In: GIES-STUBER, Petra; BLECKING, Diethelm (Eds.). Sport-Integration-Europe. Widening horizons in intercultural education. Baltmannsweiler: Schneider, 2008. p. 36-46.

GRIMMINGER, Elke. Intercultural competence among sports and PE teachers. Theoretical foundations and empirical verification. European Journal of Teacher Education, Abingdon, v. 34, n. 3, p. 317-331, 2011. 
JOKIKOKKO, Katri. Interculturally trained Finnish teachers'conceptions of diversity and intercultural competence. Intercultural Education, Abingdon, v. 16, n. 1, p. 69-83, 2005.

LEIVA OLIVENCIA, Juan José. Interculturalidad, gestión de la convivencia y diversidad cultural en la escuela: Un estudio de actitudes del profesorado. Revista Iberoamericana de Educación, Madrid, v. 46, n. 2, p. 1-14, 2008. Disponible en: <http://www.rieoei.org/deloslectores/2297Leivav2. pdf >. Acceso: 19 ago. 2015.

LÓPEZ PASTOR, Víctor. Didáctica de la educación física, desigualdad y transformación social. Estudios Pedagógicos, Valdivia, n. 38, p. 155-176, 2012.

LÓPEZ, Víctor; PÉREZ, Ángel; MONJAS, Roberto. La atención a la diversidad en el área de Educación Física. La integración del alumnado con necesidades educativas específicas, especialmente el alumnado inmigrante y de minorías étnicas. Lectura: Educación Física y Deportes, Buenos Aires, n. 106, 2007. Disponible en:. http://www.efdeportes.com/efd106/laatencion-a-la-diversidad-en-educacion-fisica.htm. Acceso en: 9 out. 2015.

MURUA-CARTON, Hilario; ETXEBERRIA, Félix; GARMENDIA, Joxe; ARRIETA, Elisabet. ¿Qué otras competencias debe tener el profesorado del alumnado inmigrante?, Magis, Revista Internacional de Investigación en Educación, Bogotá, v. 5, n. 10, p. 109-132, 2012. Disponible en: <http://www.redalyc.org/pdf/2810/281024896007.pdf >. Acceso en: 13 ago. 2015.

PARICIO, María. Dimensión intercultural en la enseñanza de las lenguas y formación del profesorado. Revista iberoamericana de educación, Madrid, v. 34, n. 4, 2004. Disponible en: $<$ http://www.ugr.es/ portalin/articulos/PL numero21/14\%20\%20Silvina.pdf>. Acceso en: 13 ago. 2015.

PERRY, Laura; SOUTHWELL, Leonie. Developing intercultural understanding and skills: models and approaches. Intercultural Education, Abingdon, v. 22, n. 6, p. 453-466, 2011. Disponible en: <http://dx.doi.org/10.1080/14675986.2011.644948>. Acceso en: 13 ago.2015.

PINYA, Carme; MUNTANER, Joan Jordi. La interculturalidad en la formación permanente del profesorado. Diálogos: Educación y formación de personas adultas, Barcelona, v. 3, n. p. 79-80, p. 34-42, 2014.

RUIZ VALDIVIA, Macarena; MOLERO-LÓPEZ, David; ZAGALAZ, María; CACHÓN, Javier. Análisis de la integración del alumnado inmigrante a través de las clases de Educación Física. Apuntes. Educación Física y Deportes, Barcelona, v. 182, n. p. 26-34, 2012. Disponible en: <http://dx.doi.org/10.5672/apunts.2014-0983.es.(2012/2).108.03>. Acceso en: 10 jun. 2015.

SALES, Auxiliadora. La formación intercultural del profesorado: estrategias para un proceso de investigación acción. Educatio Siglo XXI, v. 30, n. 1, p. 113-132, 2012. Disponible en: <http:// revistas.um.es/educatio/article/view/149171>. Acceso en: 10 jun. 2015.

TAROZZI, Massimiliano. Building an 'intercultural ethos' in teacher education. Intercultural Education, Abingdon, v. 25, n. 2, p. 128-142, 2014.

UNESCO. Informe Mundial. Invertir en la diversidad cultural y el diálogo intercultural. Organización de las Naciones Unidas para la Educación, la Ciencia y la Cultura. París: UNESCO, 2009. Disponible en: <http://unesdoc.unesco.org/images/0018/001878/187828s.pdf>. Acceso en: 30 jun. 2015.

VALERO, Alfonso; GÓMEZ, Manuel. Multiculturalidad en las clases de Educación Física a través de los juegos del mundo. Lectura: Educación Física y Deportes, Buenos Aires, n. 105, 2007, Disponible en: <http://www.efdeportes.com/efd105/multiculturalidad-en-las-clases-de-educacionfisica-a-traves-de-los-juegos-del-mundo.htm>. Acceso en: : 26 jun. 2015. 